\title{
High quality factor GaAs microcavity with buried bullseye defects
}

Winkler, K.; Gregersen, Niels; Hayrynen, T.; Bradel, B.; Schade, A.; Emmerling, M.; Kamp, M.; Höfling, S.; Schneider, C.

Published in:

Physical Review Materials

Link to article, DOI:

10.1103/PhysRevMaterials.2.052201

Publication date:

2018

Document Version

Publisher's PDF, also known as Version of record

Link back to DTU Orbit

Citation (APA):

Winkler, K., Gregersen, N., Hayrynen, T., Bradel, B., Schade, A., Emmerling, M., Kamp, M., Höfling, S., \& Schneider, C. (2018). High quality factor GaAs microcavity with buried bullseye defects. Physical Review Materials, 2(5), [052201 ]. https://doi.org/10.1103/PhysRevMaterials.2.052201

\section{General rights}

Copyright and moral rights for the publications made accessible in the public portal are retained by the authors and/or other copyright owners and it is a condition of accessing publications that users recognise and abide by the legal requirements associated with these rights.

- Users may download and print one copy of any publication from the public portal for the purpose of private study or research.

- You may not further distribute the material or use it for any profit-making activity or commercial gain

- You may freely distribute the URL identifying the publication in the public portal 


\title{
High quality factor GaAs microcavity with buried bullseye defects
}

\author{
K. Winkler, ${ }^{1}$ N. Gregersen, ${ }^{2}$ T. Häyrynen, ${ }^{2}$ B. Bradel,${ }^{1}$ A. Schade, ${ }^{1}$ M. Emmerling, ${ }^{1}$ M. Kamp, ${ }^{1}$ S. Höfling,,${ }^{1,3}$ and C. Schneider ${ }^{1}$ \\ ${ }^{1}$ Technische Physik, Wilhelm-Conrad-Röntgen-Research Center for Complex Material Systems, \\ Universität Würzburg, Am Hubland, D-97074 Würzburg, Germany \\ ${ }^{2}$ DTU Fotonik, Department of Photonics Engineering, Technical University of Denmark, Ørsteds Plads, \\ Building 343, DK-2800 Kongens Lyngby, Denmark \\ ${ }^{3}$ SUPA, School of Physics and Astronomy, University of St Andrews, St Andrews KY16 9SS, United Kingdom
}

(Received 23 November 2017; revised manuscript received 14 March 2018; published 17 May 2018)

\begin{abstract}
The development of high quality factor solid-state microcavities with low mode volumes has paved the way towards on-chip cavity quantum electrodynamics experiments and the development of high-performance nanophotonic devices. Here, we report on the implementation of a new kind of solid-state vertical microcavity, which allows for confinement of the electromagnetic field in the lateral direction without deep etching. The confinement originates from a local elongation of the cavity layer imprinted in a shallow etch and epitaxial overgrowth technique. We show that it is possible to improve the quality factor of such microcavities by a specific in-plane bullseye geometry consisting of a set of concentric rings with subwavelength dimensions. This design results in a smooth effective lateral photonic potential and therefore in a reduction of lateral scattering losses, which makes it highly appealing for experiments in the framework of exciton-polariton physics demanding tight spatial confinement.
\end{abstract}

DOI: 10.1103/PhysRevMaterials.2.052201

\section{INTRODUCTION}

Tailoring the photonic confinement in a microcavity environment is a key element of fundamental light-matter studies. This confinement can be achieved by different technological approaches, such as patterning of microcavity pillars $[1,2]$, photonic crystal cavities [3,4], microdisks [5], and toroidal microcavities [6]. The interaction of the resulting zero-dimensional (0D) modes with an optically active matter part, such as quantum dots (QDs) or quantum wells, is the foundation of various devices, including nanophotonic lasers [7], solid-state single-photon sources [8-10], spin-photon interfaces [11], and platforms for polariton condensation [12]. In these systems, it is desirable to achieve small mode volumes through strong 3D mode confinement to increase the lightmatter interaction while minimizing detrimental effects on the particle lifetime, such as scattering losses due to the roughness of semiconductor-air interfaces or coupling into leaky modes. In principle, it has been shown in various implementations, including the micropillar and the photonic crystal geometry, that photonic designs relying on gently shaping the confinement of light are superior to solid-box types of microcavities [13-15]. However, most cavity architectures described above rely on deep etching to provide a strong lateral confinement, which is detrimental for many experiments with extended high quality 2D quantum wells, such as in the framework of excitonpolariton physics [12]. There, it has been shown that deep etching through the active materials leads to a strong degradation of the signal via the emergence of a broad, uncoupled background luminescence from excitons at the etched surfaces [16,17]. This effect is one of the most significant obstacles in the field of genuine quantum polaritonics, and has put up extreme challenges for a variety of important observations [18,19]. In addition, it has been repeatedly reported that the required deep etching in conventional micropillar and photonic crystal cavities typically leads to a deterioration of the performance of quantum dot single-photon sources regarding the coherence of the emitted photons [20,21], as well as QD spin-photon interfaces regarding the dephasing of the QD spin [22]. In this spirit, cavities have been developed which host a buried optical defect which alters the photonic potential by a local elongation of the cavity [23]. In such structures, the trapping potential is well tunable [24], which generally yields a large flexibility to tailor the photonic potential landscape [16]. However, similar to the case of a deeply etched micropillar, buried mesa cavities are also subject to in-plane scattering which ultimately limits the available cavity quality $(Q)$ factors in the regime of tight photonic confinement. Recently, it was shown [25] that a photonic potential that mimics a Gaussian distribution in such a device can reduce in-plane scattering losses and increase the quality factor by two orders of magnitude. Recent realizations [26,27] of such defects rely either on random distribution [27] or on time-consuming patterning techniques which are not fully compatible with highest quality III-V quantum wells [26].

In this Rapid Communication, we introduce a cavity design which enables a smooth lateral optical confinement in a distributed Bragg reflector (DBR) type of structure, which can be defined by scalable electron beam lithography and is fully compatible with GaAs-based molecular beam epitaxy. In Sec. II we will present the fabrication details of our investigated devices. Details of the modeling are given in Sec. III, followed by a discussion of the experimental and theoretical findings in Sec. IV.

\section{FABRICATION}

A sketch of the vertical microcavity sample investigated in this Rapid Communication is schematically shown in Fig. 1(a) 
(a)

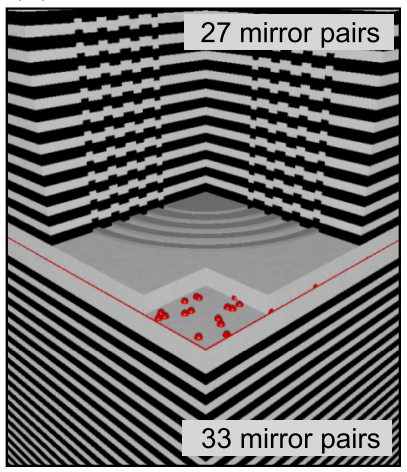

(b)

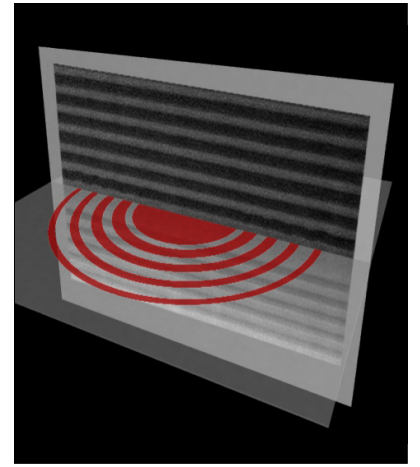

(c)
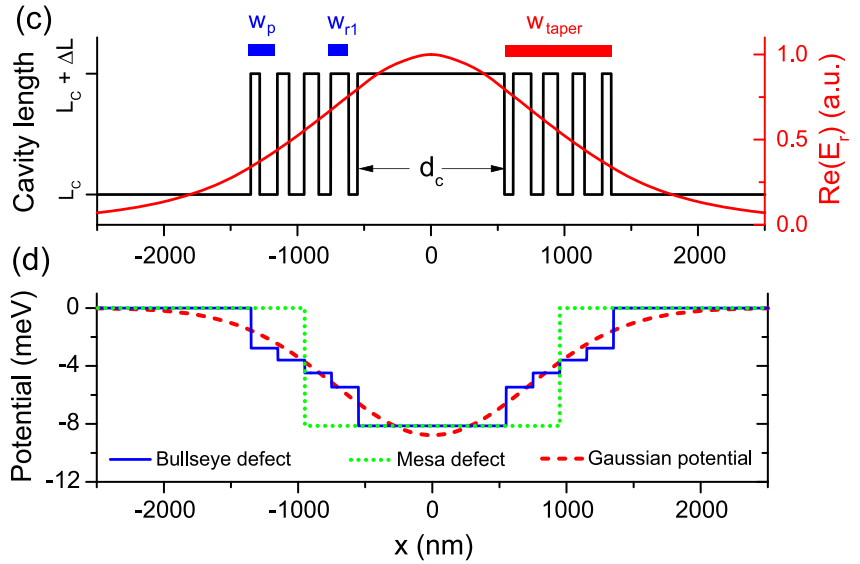

FIG. 1. (a) Sketch of the vertical AlAs/GaAs microcavity sample with a buried bullseye defect. InAs quantum dots at the center of the $\lambda$ cavity are red color coded and serve as an internal light source. (b) Cross-sectional SEM image from a cut through the sample at the center of the bullseye with $d_{c}=1.1 \mu \mathrm{m}$ and (c) corresponding nominal profile of the local elongation of the cavity layer in respect to the surrounding. Simulated real part $\operatorname{Re}\left(E_{r}\right)$ of the radial mode profile at the vertical position of the quantum dot layer (red line). (d) Averaged shape of the cross-sectional potential of the bullseye (blue line) and mesa defect (green dotted line) which provides the same confinement depth for the fundamental optical mode. A Gaussian-shaped potential is plotted as a guide to the eye (red dotted line).

by a cut through the $\frac{\lambda}{n}$-thick cavity layer ( $n$ is the refractive index of the material). The fabrication process consists of an etch-and-overgrowth routine: The bottom mirror (33 AlAs/GaAs DBR layers) and the cavity layer with embedded InAs quantum dots (shown in red) are epitaxially grown on a doped 3-in. GaAs wafer. This wafer is then patterned via optical and electron beam lithography and wet chemical etching to define the vertically elongated defects on top of the cavity layer with a height of $\Delta L \approx 5 \mathrm{~nm}$. After finalizing the sample by epitaxial overgrowth with $27 \mathrm{AlAs} / \mathrm{GaAs}$ DBR mirror pairs, well-defined optical traps are realized in this way due to the cavity resonance shift caused by the elongation. Here, it is important to note that the wet chemical etching in contrast to plasma etching is crucial in order to keep the amount of crystal damage to the optical active media minimal. To mimic a smooth lateral confinement, in order to suppress lateral scattering, we extended single mesa defects of circular lateral shape with diameter $d_{c}$ by surrounding rings [see Fig. 1(c) for a cross-sectional cavity layer height profile], resembling a bullseye structure. As shown in Fig. 1(c), the thicknesses of the rings are much smaller than the extent of the computed mode profile, and the cavity mode thus experiences a tapered reduction of the effective potential depth outwards. Figure 1(b) shows a scanning electron microscope (SEM) image of such a tapered bullseye defect, which was sliced through the center by an focused ion beam overlayed with the nominal trap geometry (red) as a guide to the eye.

While the taper period width $w_{p}$ is constant for each of the four ring segments, the width of the rings $w_{r}$ is successively reduced outwards. Thus we can define a filling factor of $F_{s}=w_{r_{s}} / w_{p}$ for each individual segment $s$. To describe the cross-sectional potential depth generated by the taper, we estimate the averaged effective height $F_{s} * \Delta L$ for each segment and calculate the photonic vertical resonance through transfer-matrix simulations. Figure 1(d) shows the calculated potential for a tapered defect with $d_{c}=1.1 \mu \mathrm{m}$ in comparison to a circular mesa defect with diameter $d=1.9 \mu \mathrm{m}$, revealing that in case of a tapered defect, the potential approaches a Gaussian shape. Here, we study several bullseye defects, with $d_{c}$ ranging from 0.5 to $5.0 \mu \mathrm{m}$, each surrounded by four taper segments with period $w_{p}=200 \mathrm{~nm}$ and ring width of $w_{r_{1}}=134 \mathrm{~nm}$ to $w_{r_{4}}=68 \mathrm{~nm}$. These buried bullseye defects are patterned repeatedly over the cavity sample, together with single mesa defects where $d$ is ranging from 0.5 to $10 \mu \mathrm{m}$, to enable a direct comparison between both photonic trapping geometries.

\section{MODELING}

We use a recently introduced a numerical technique based on an open geometry formulation of the Fourier modal method $[28,29]$. Here, the geometry is divided into material sections uniform along the propagation $z$ axis. A complete vectorial description is used to describe the Maxwell's equations in the $z$ invariant material section and compute the lateral eigenmodes. Using cylindrical coordinates in the rotationally symmetric case simplifies the problem to a 1D expansion in the radial coordinate. The $z$ dependence is treated by combining $z$ invariant sections using the scattering matrix formalism [30].

To determine the $Q$ of the cavity mode of the buried defect trap, we consider the frequency-dependent reflectivity matrices $\overline{\overline{R_{T}}}$ and $\overline{\overline{R_{B}}}$ describing the reflection of the cavity eigenmodes at the cavity-DBR interfaces. We then solve the eigenvalue problem

$$
\overline{\overline{R_{T} P R_{B} P}} \bar{c}=R_{r} \bar{c},
$$

where $\overline{\bar{P}}$ is the cavity propagation matrix.

We obtain an eigenvector $\bar{c}$ and an eigenvalue $R_{r}$ for the cavity mode, and we determine the resonance wavelength $\lambda_{r}=2 \pi c / \omega_{r}$ by requiring that $\arg \left(R_{r}\right)=0$, where $\arg$ is the argument of the complex value $R_{r}$. The $Q$ is then obtained from the expression [31]

$$
Q=\frac{-\lambda}{2\left(1-\left|R_{r}\right|\right)} \frac{\partial}{\partial \lambda} \arg \left(R_{r}\right) .
$$

A significant technical difficulty lies in the determination of the eigenvector representing the cavity mode. Unlike a 
pillar microcavity, where the eigenvector of the fundamental cavity mode [31] is dominated by the fundamental $\mathrm{HE}_{11}$ mode contribution, the planar cavity eigenvector has no similar characteristic signature. To identify the eigenvector, we thus first compute the total local density of states at the QD position. After having identified the spectral resonance peak, we then expand [32] the field on the eigenvectors of the round-trip matrix obtained from Eq. (1) at the resonance frequency. The cavity mode eigenvector is then identified as the dominating contribution in this expansion.

\section{RESULTS AND DISCUSSION}

To investigate the influence of the bullseye-shaped cavity on the optical mode pattern, we performed low-temperature angular-resolved photoluminescence (PL) measurements via nonresonant injection with a tunable Ti:sapphire laser. The photonic modes are illuminated via nonresonant quantum dot-cavity coupling by the optical active quantum dot ensemble with an ensemble linewidth of $26 \mathrm{meV}$ and a central emission energy of $1.302 \mathrm{eV}$, which is spectrally matched to the photonic resonances of the microcavity. We acquire the full mode pattern in momentum space in a photoluminescence setup utilizing the back Fourier plane imaging method [33]. In Fig. 2 we point out differences of the spectral mode distribution between a mesa defect [Fig. 2(a)] and a bullseye defect [Fig. 2(b)]: As the taper rings alter the effective lateral size of the trapping potential in the bullseye, the central diameter of the traps is no longer a good parameter for comparison. Instead, we will compare defects which feature an identical energy difference between the ground mode and the barrier energy minimum. The barrier energy can be conveniently measured in our cavities as it appears as a characteristic parabolic dispersion of finite mass photons on the high-energy side of the confined modes (not shown in Fig. 2). A comparable confinement depth is found when $d_{\mathrm{eff}}=d_{c}+800 \mathrm{~nm}$ of bullseye defects equals $d$ of conventional defects throughout the given diameter variation in our sample.

In Fig. 2 we depict two exemplary angular-resolved photoluminescence spectra of a mesa defect and a bullseye defect cavity with a diameter of $d_{\text {eff }} \approx 5.0 \mu \mathrm{m}$. In order to enhance the comparability in this study, we have normalized the energy axis to the minimum of the continuous photonic dispersion which stems from the planar surrounding cavity. Both the conventional mesa defect [Fig. 2(a)] and the bullseye defect [Fig. 2(b)] lead to a clear discretization of the optical resonances. The circular geometry of the system allows one to characterize the emerging resonances by the radial and orbital quantum numbers $n, m$ [34]. For the case of the bullseye defect cavity, we find two significant modifications of the mode spectrum. First, the energetic difference between the third and fourth modes as well as between the fifth and sixth modes is significantly reduced, as compared to the mesa cavity. This deviation results from a effectively more hemispherical shape of the confinement potential, where the mode energies follow a Laguerre-Gauss description instead of a Bessel-Gauss description. It is worth noting that for an ideal hemispheric potential, these modes would be fully degenerate. Another result is a generally more equidistant mode distribution, as seen in Fig. 2(d) compared to Fig. 2(d), emerging from the almost
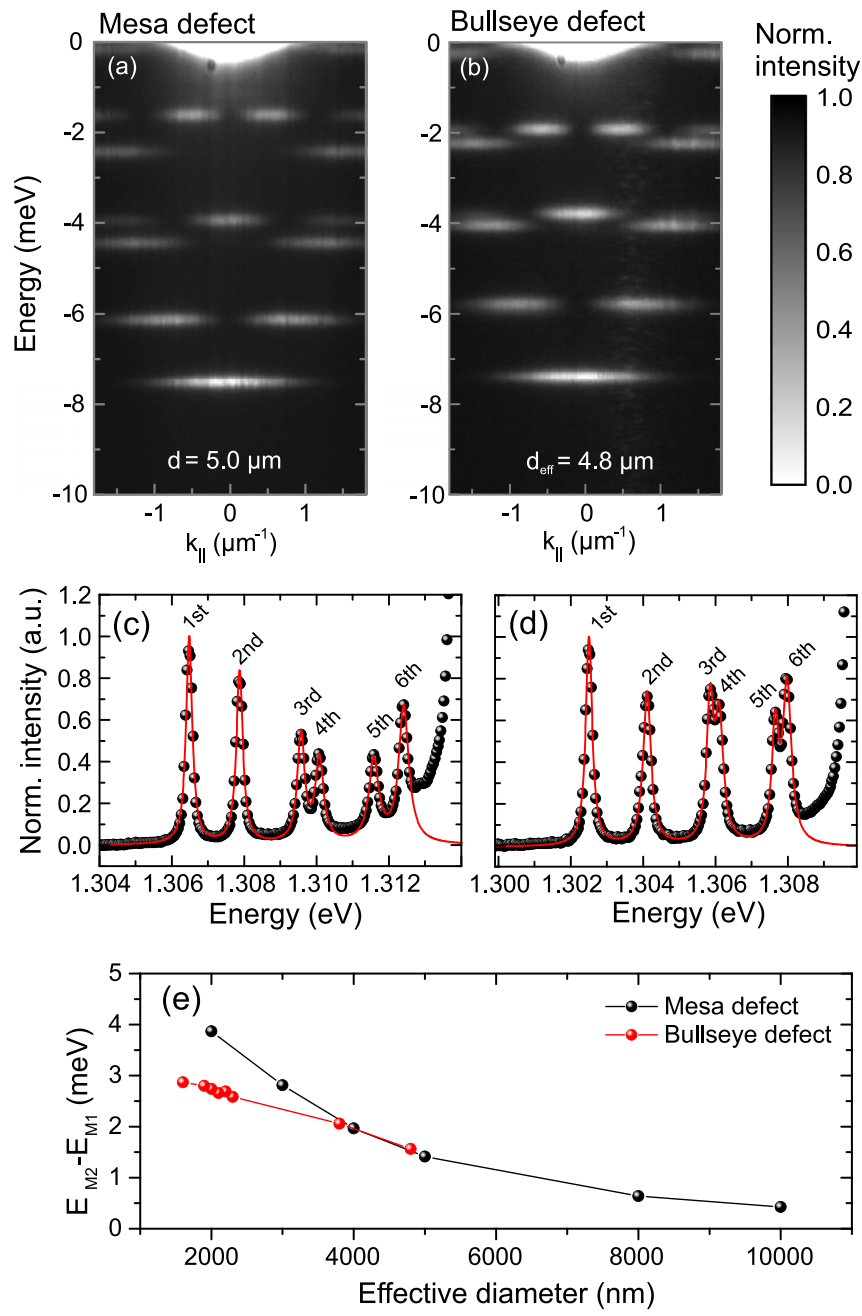

FIG. 2. Momentum-resolved photoluminescence spectra of confined states in mesa defects (a) and bullseye defects (b). The energy axis is given with respect to the minimum of the parabolic dispersion stemming from the surrounding barrier region that amounts to 1.314 and $1.310 \mathrm{eV}$, respectively. Line profiles of mode distribution shown in (c) and (d) correspond to (a) and (b), respectively, and are integrated over $k$ space. (e) Intermode energy distance between first and second confined modes compared for bullseye and mesa defects.

Gaussian shape of the potential. These spectra were acquired by binning over the full $k$ range in Figs. 2(a) and 2(b).

As the diameter of the cavities is reduced, we note that the second mode $(n=1, m=1)$ is slightly redshifted for our bullseye cavities with respect to the mesa defect cavity [see Fig. 2(e) for a comparison of intermode energy distance]. In fact, this behavior can be expected as the confinement potential becomes more Gaussian shaped. Consequently, the influence of the altered potential in the bullseye defects should be most prominent for defects ranging from $d_{\mathrm{eff}} \approx 1$ to $4 \mu \mathrm{m}$.

In order to study the capability of our cavities to store the electromagnetic field, we investigate the quality factor of these defects on a photoluminescence setup at cryogenic temperatures of $15 \mathrm{~K}$ under nonresonant excitation at $532 \mathrm{~nm}$. The laser power of $6 \mu \mathrm{W}$ lies well within the weak pumping regime. From the Lorentzian-shaped emission peaks we extracted the full width at half maximum height $\Delta \lambda$ and emission 

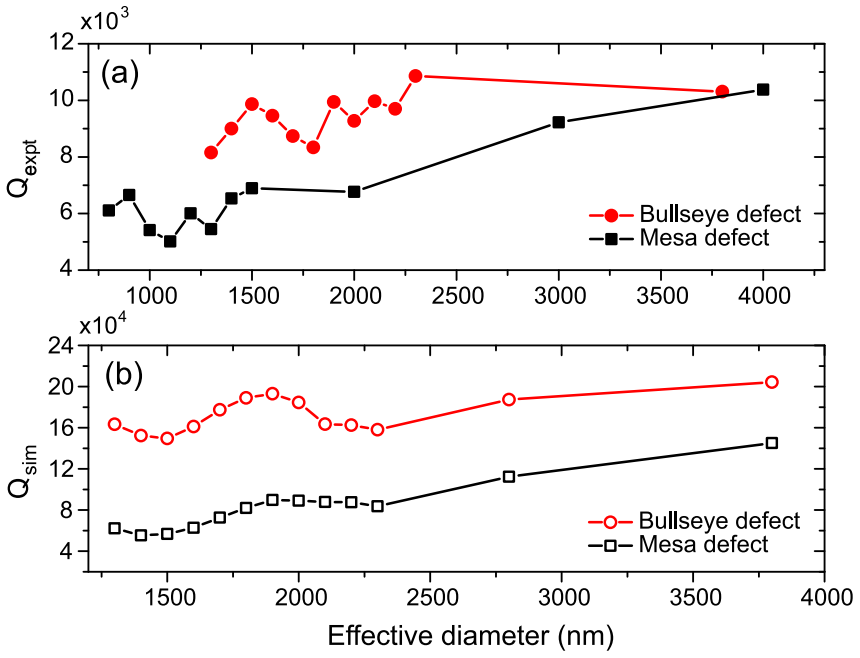

FIG. 3. Quality factor comparison of bullseye and mesa defects. (a) $Q_{\text {expt }}$ calculated from low-temperature PL measurements and (b) $Q_{\text {sim }}$ calculated by numerical modeling.

wavelength $\lambda$ to calculate the quality factor $Q_{\text {expt }}=\Delta \lambda / \lambda$ of the fundamental mode. A comparison of the highest measured quality factors from bullseye defects and conventional mesa defects of varying diameter is shown in Fig. 3(a), whereas we plot the corresponding calculated $Q_{\text {sim }}$ in Fig. 3(b). Both in our experimental and theoretical investigation we observe a clear increase of the $Q$ in the bullseye geometry, which outperforms the mesa defects approximately by a factor of 1.3 (experimental values) to 2.0 (theoretical values).

Our theoretical study outlines that a microcavity resonance with $Q>100000$ can be realized with a bullseye defect even for effective diameters less than $1.5 \mu \mathrm{m}$, in contrast to a mesa defect. We note that our experimental values for $Q$ are reduced by roughly one order of magnitude compared to the theoretical values. This reduction-typical for experimental realizations of high- $Q$ designs $[13,31,35]$ —results most likely from material absorption or remaining structural imperfections not accounted for in the calculations.

To analyze the potential for enhanced light-matter interaction strength, we present in Fig. 4(a) computed mode volumes for the investigated devices as a function of the effective diameter. Importantly, the mode volumes of the mesa and bullseye defects are very similar. In the small diameter regime, the photonic potential well width becomes too small to support a confined mode, and an increase in mode volume with decreasing diameter for both defect types is observed. Similarly to the case of adiabatic micropillar cavities [13], this overall behavior is expected as the tapered bullseye geometry serves to increase the $Q$ factor while leaving the mode volume almost unchanged.

In order to provide a fair comparison between our system and a more traditional cavity implementation, we plot a typical microcavity pillar mode volume-diameter dependency [36] in Fig. 4(a). As the diameter increases, we observe that the relative mode volume deviation between the defects and the micropillar geometries is continuously reduced. However, the defect mode volumes in the small diameter regime are significantly larger due to the lack of hard wall lateral confinement present in
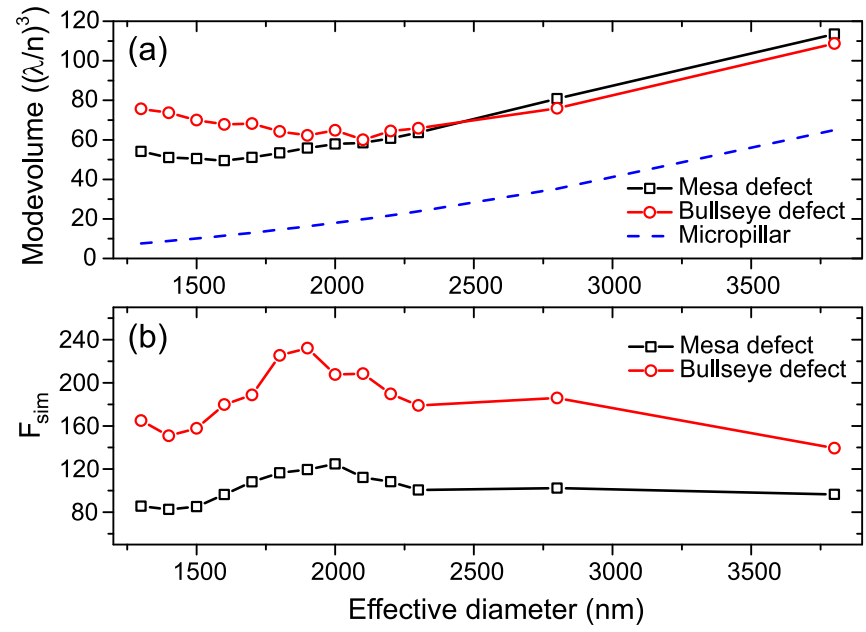

FIG. 4. (a) Normalized mode volume $V_{n}$ of bullseye and mesa defects. A typical theoretical dependency $V=c d^{2}$ with $c=$ $4.5(\lambda / n)^{3} \mu \mathrm{m}^{-2}$ [36] for conventional micropillars is shown as a blue dashed line. (b) Purcell enhancement calculated from $Q_{\text {sim }}$ and $V_{n}$ of bullseye and mesa defects.

the micropillar geometry. This increased mode volume of the defect geometries is the price to pay for a smooth lateral confinement. However, we want to emphasize that the mode volume can be decreased in a lateral extent [25] by a deeper etch depth due to the resulting higher potential, which composes an interesting design problem beyond the scope of this Rapid Communication. The numerically calculated mode volumes allow us to derive $F_{\text {sim }}$ for the defect geometries in Fig. 4(b). In spite of the relatively large mode volume, we observe that the computed Purcell enhancement $F_{\text {sim }}=3 Q_{\text {sim }} /\left(4 \pi^{2} V_{n}\right)$ for the bullseye geometry can still exceed 240 for an optimum effective diameter of $\approx 1800 \mathrm{~nm}$. This puts our device platform in the central focus of advanced light-matter coupling in the solid state.

\section{CONCLUSION}

In conclusion, we have designed and implemented a cavity architecture, which in principle allows one to squeeze optical modes of high quality factor into very small volumes in a monolithic III-V microcavity. Uniquely, our approach does not rely on deep reactive ion etching, which makes it highly suitable for experiments relying on highest quality solid-state emitters. One field where we anticipate that our microcavity could be of great use is experiments relying on microcavity quantum well exciton polaritons [12] with ultratight confinement as the bullseye defect concept can be readily combined with an embedded quantum well. The hybrid light-matter character of these quasiparticles leads to a density-dependent particle interaction which can be utilized for the generation of single quantum states of polaritons $[18,19]$ in a scalable and uniform manner. Up to now, the realization of such a polariton blockade device is still elusive because a spectrally narrow resonance comparable to the interaction blueshift per particle is needed, together with a very small system cross section in the submicron regime which is not fulfilled by III-V cavities thus far. 
A related field where we believe that our cavities could be beneficial is the design of high-performance quantum dot single-photon sources. As we show in our theoretical calculations, the bullseye microcavities can support ultrahigh Purcell factors and should be well suited to implement highbrightness, high-speed quantum dot single photons with the largest indistinguishability.

\section{ACKNOWLEDGMENTS}

The authors acknowledge financial support from the State of Bavaria, as well as from the DFG within the Project Schn1376/3.1: Polariton based single photon sources, and from the Independent Research Fund Denmark Technology and Production Sciences (Sapere Aude LOQIT, DFF-4005-00370).
[1] Single Quantum Dots, edited by J. M. Gerard and P. Michler (Springer, Berlin, 2003).

[2] C. Schneider, P. Gold, S. Reitzenstein, S. Höfling, and M. Kamp, Appl. Phys. B 122, 1 (2016).

[3] S. John, Phys. Rev. Lett. 58, 2486 (1987).

[4] E. Yablonovitch, Phys. Rev. Lett. 58, 2059 (1987).

[5] B. Gayral, J. M. Gérard, A. Lemaître, C. Dupuis, L. Manin, and J. L. Pelouard, Appl. Phys. Lett. 75, 1908 (1999).

[6] D. K. Armani, T. J. Kippenberg, S. M. Spillane, and K. J. Vahala, Nature (London) 421, 925 (2003).

[7] S. Strauf and F. Jahnke, Laser Photon. Rev. 5, 607 (2011).

[8] W. L. Barnes, G. Björk, J. M. Gérard, P. Jonsson, J. A. E. Wasey, P. T. Worthing, and V. Zwiller, Eur. Phys. J. D 18, 197 (2002).

[9] T. Heindel, C. Schneider, M. Lermer, S. H. Kwon, T. Braun, S. Reitzenstein, S. Höfling, M. Kamp, and A. Forchel, Appl. Phys. Lett. 96, 011107 (2010).

[10] Y.-M. He, J. Liu, S. Maier, M. Emmerling, S. Gerhardt, M. Davanço, K. Srinivasan, C. Schneider, and S. Höfling, Optica 4, 802 (2017).

[11] A. B. Young, R. Oulton, C. Y. Hu, A. C. T. Thijssen, C. Schneider, S. Reitzenstein, M. Kamp, S. Höfling, L. Worschech, A. Forchel, and J. G. Rarity, Phys. Rev. A 84, 011803 (2011).

[12] A. V. Kavokin, J. J. Baumberg, G. Malpuech, and F. Laussy, Microcavities (Oxford University Press, Oxford, U.K., 2006).

[13] M. Lermer, N. Gregersen, F. Dunzer, S. Reitzenstein, S. Höfling, J. Mørk, L. Worschech, M. Kamp, and A. Forchel, Phys. Rev. Lett. 108, 057402 (2012).

[14] Y. Akahane, T. Asano, B.-S. Song, and S. Noda, Nature (London) 425, 944 (2003).

[15] K. Srinivasan and O. Painter, Opt. Express 10, 670 (2002).

[16] C. Schneider, K. Winkler, M. D. Fraser, M. Kamp, Y. Yamamoto, E. A. Ostrovskaya, and S. Höfling, Rep. Prog. Phys. 80, 016503 (2017).

[17] D. Bajoni, P. Senellart, E. Wertz, I. Sagnes, A. Miard, A. Lemaître, and J. Bloch, Phys. Rev. Lett. 100, 047401 (2008).

[18] A. Verger, C. Ciuti, and I. Carusotto, Phys. Rev. B 73, 193306 (2006).

[19] N. Na and Y. Yamamoto, New J. Phys. 12, 123001 (2010).
[20] J.-H. Kim, T. Cai, C. J. K. Richardson, R. P. Leavitt, and E. Waks, Optica 3, 577 (2016).

[21] O. Gazzano, S. Michaelis de Vasconcellos, C. Arnold, A. Nowak, E. Galopin, I. Sagnes, L. Lanco, A. Lemaître, and P. Senellart, Nat. Commun. 4, 1425 (2013).

[22] D. Press, K. De Greve, P. L. McMahon, T. D. Ladd, B. Friess, C. Schneider, M. Kamp, S. Höfling, A. Forchel, and Y. Yamamoto, Nat. Photonics 4, 367 (2010).

[23] O. El Daïf, A. Baas, T. Guillet, J.-P. Brantut, R. I. Kaitouni, J. L. Staehli, F. Morier-Genoud, and B. Deveaud, Appl. Phys. Lett. 88, 061105 (2006).

[24] K. Winkler, J. Fischer, A. Schade, M. Amthor, R. Dall, J. Geßler, M. Emmerling, E. A. Ostrovskaya, M. Kamp, C. Schneider, and S. Höfling, New J. Phys. 17, 023001 (2015).

[25] F. Ding, T. Stöferle, L. Mai, A. Knoll, and R. F. Mahrt, Phys. Rev. B 87, 161116 (2013).

[26] L. Mai, F. Ding, T. Stöferle, A. Knoll, B. J. Offrein, and R. F. Mahrt, Appl. Phys. Lett. 103, 243305 (2013).

[27] S. Maier, P. Gold, A. Forchel, N. Gregersen, J. Mørk, S. Höfling, C. Schneider, and M. Kamp, Opt. Express 22, 8136 (2014).

[28] T. Häyrynen, J. R. de Lasson, and N. Gregersen, J. Opt. Soc. Am. A 33, 1298 (2016).

[29] T. Häyrynen, A. D. Osterkryger, J. R. de Lasson, and N. Gregersen, J. Opt. Soc. Am. A 34, 1632 (2017).

[30] L. Li, J. Opt. Soc. Am. A 13, 1024 (1996).

[31] N. Gregersen, S. Reitzenstein, C. Kistner, M. Strauss, C. Schneider, S. Höfling, L. Worschech, A. Forchel, T. R. Nielsen, J. Mørk, and J.-M. Gérard, IEEE J. Quantum Electron. 46, 1470 (2010).

[32] T. Häyrynen and N. Gregersen (unpublished).

[33] C. W. Lai, N. Y. Kim, S. Utsunomiya, G. Roumpos, H. Deng, M. D. Fraser, T. Byrnes, P. Recher, N. Kumada, T. Fujisawa, and Y. Yamamoto, Nature (London) 450, 529 (2007).

[34] L. Pierre, D. Sarchi, and V. Savona. Phys. Status Solidi 3, 2428 (2006).

[35] S. Reitzenstein and A. Forchel, J. Phys. D: Appl. Phys. 43, 033001 (2010).

[36] N. Gregersen, P. Kaer, and J. Mørk, IEEE J. Sel. Top. Quantum Electron. 19, 9000516 (2013). 